\title{
Studies on the Relationship of Medical Resources and Development of Medical Advanced Education
}

\author{
Na Wang \\ Department of Anesthesiology \\ The First Hospital of Jilin University \\ Changchun, China \\ wangna080613@163.com
}

\author{
Jinguo Wang *(corresponding author) \\ Department of Urology \\ The First Hospital of Jilin University \\ Changchun, China \\ wangjinguolily@163.com
}

\begin{abstract}
To put forward the development strategy to speed up the talents cultivating. Cultivated talents are very important strategic resources in the development of college. Gathering and continuing to cultivate high-quality talents is necessary. In terms of college, that is the increase of the competitiveness and in terms of employees, that is the improvement of life quality and life satisfaction. Talent resources are the first resource in the development of college. The college system reform which brings opportunity and challenge must adhere to give priority to human resources development.
\end{abstract}

Keywords-Medical resources, medical advanced education, balance

\section{INTRODUCTION}

Human resources for health includes: (1) had been working in the health services in the industry of health personnel, such as doctors, nurses, etc.; (2) the future of health workers, such as medical college students; (3) potential health workers, they are according to the plan and needed can be engaged in special vocational and training of health personnel, such as sanitary equipment maintenance personnel, health, education and medical information processing personnel, etc.

\section{MedicAl Resource AND MEdicAl EduCATION}

Widespread around the world "health manpower imbalance" phenomenon, the form mainly has two aspects: one is the quantity and quality of imbalance, including amount, type, distribution, and quality (such as service quality and service quality). The second is the country, the residents of health service demand and utilization of imbalance between ability, support and maintenance. Therefore, the present situation of human resources for health, planning, forecasting and management research has become one of hot topics. Research on human resources for health, its purpose is to better from quantity, quality and distribution adjustment on the existing stock, optimize increment, and make it reach the purpose of to mobilize human resources for health subjective initiative, in order to promote the process of the health development [1].

Foreign health manpower needs research began in the early 1960s. The United States in 1972 to its specialist demand forecasting, the results showed that in 1990 the United States will have 70000 more than demand, and by 2000 there will be
2000 physicians exceeds the demand. In general, foreign scholars studies in total health manpower forecasting is less, some mainly focused on the level of specialized subject doctor forecasts of volume with pricing.

\section{A. Health resources are the decisive factor in the} development of health service.

Human resources for health research in China start late, but develop fast. In recent 10 years, many scholars in our country much health manpower forecasting method, and the regional health workforce of our country exploratory forecast, and achieved initial results, for the public health administrative department formulate long-term human development planning provides certain help. Medical institutions of higher education is the main supply of human resources for health channel, the cultivation of medical talents quality will directly affect the level of community health service, to the national health and social stability. The development of higher medical education, to a country and society is of great significance [2]. Therefore, analyze the present situation of higher medical education research of higher medical education and health human resources supply and demand balance, to health talented person development strategy has important practical significance.

In order to effectively control the scale of the enrollment of medical colleges and universities, in order to guarantee the quality of medical talents and the balance between supply and demand, the European and American countries mainly through the government's macroeconomic regulation and control, for example, the federal government, according to the supply and demand situation of medical graduates, by adding or funding cuts to control the scale of the enrollment of medical college [3]. France, medical colleges' exam out of proportion is the government according to the residents and doctors with the ratio of the total population after measure. The UK by the ministry of education and the National Health Service is the department of funding for each medical college scale to carry on the strict macroeconomic control.

B. The reasonable structure, the balanced distribution, scientific management of health resources all contribute to not only the development of health service, but also economy and society.

Medical institutions of higher education in China have a larger development, for our country public transport a large 
number of qualified medical talents. Not only have the clear direction of running, but the medical professional setting is more complete, the system also tend to be more reasonable, through practice gradually explore the rule of medical education, and have the discipline characteristic, formed the medical education management system and operational mechanism, initially established the basic education, including school education after graduation from the continuum of the continuing education of medical education system [4]. Medical education scale, quality, efficiency has improved obviously. Number of medical colleges and universities and the number of students are now taking shape than in the early days after foundation has greatly improved. Strengthening medical education teachers, teaching conditions gradually improve. Before the splendid performance, the analysis of current medical higher education, there are still some aspects worthy of our thinking.

The configuration and development of medical higher education resources and the realization of the medical and health personnel needs medical higher education has made great development, as the public transport a large number of qualified medical talents, made great contributions to the economic and social development. But on the cultivating model structure, with the requirements of the health reform and development are still out of sync. Current medical higher education scale, level, the allocation of resources and development speed, is often in reference to the national overall medical health talented person's demand, coupled with the employment of graduates. Ignore the regional economy, culture, social development level of medicine and health care personnel demand difference. It is a failure to closely combine with the medical and health care market demand of china to develop the rational and scientific development plan and talent training goal [5]. 11th five-year plan" planning for health talented person, points out that carrying out the strategy of the medical personnel, strive to build a high quality team of medical personnel, is the basic task of china medical personnel working in the new century. The optimal allocation of human resources for health is the starting point and the foothold of health talent strategy and higher medical education is a basic guarantee the high quality talent team construction of health. Therefore, the current situation of human resources for health and medical institutions of higher education is a comprehensive and objective analysis, and forecast of the demand for science in the short, medium, is advantageous to the correct view of reality, long-term scientific planning, promote the optimized allocation of human resources for health and healthy development of higher medical education, to better complete the basic task of medical personnel work.

\section{Medical institutions is the main channels of talent supply of human resources, so the quality of education will directly affect the level of community health service, the national health and social stability.}

To discuss the contradiction between supply and demand of human resources for health and medical institutions of higher education, the main reasons for health information feedback and the role of talent demand to medical education in the concrete practice of the development and reform, optimize the distribution of medical education resources, guide the rational use of medical education resources, to strengthen and promote the employment of medical students, put forward human resources for health and medical institutions of higher education development planning of scientific advice. In the end, for government decision-making departments in formulating health workforce development planning and medical higher education development planning provides valuable scientific evidence, a target allocation of health resources, make the limited health resources to exert maximum efficiency, to better service to human health.

The level of higher education structure mainly includes: structure, type structure, form structure, management system structure, distribution structure, class (professional) structure, financial structure, faculty structure, structure, teaching structure and other 10 kinds of students. The top five, usually in the country or region, within the scope of research can be referred to as the macro structure, and then five, often in a school within the scope of analysis, can be called the microstructure. This research mainly involves the hierarchical structure and professional structure.

Through review the related literature, access to the current situation of human resources for health and medical institutions of higher education at home and abroad related literature survey and demand forecasting, research progress both at home and abroad, to understand the field for the purpose of this research provides idea and reference. To our country and the health human resource and higher medical education of china's policy file collection and analysis, a comprehensive understanding of the health human resources of china and the development of higher medical education and reform situation, analysis of health human resource and the supply and demand relationship between the development of higher medical education.

Supply is in a certain period of time, under certain conditions, the enterprise is willing and able to provide the number of goods or services, here refers to the number of graduates all medical colleges. Demand is in a certain period of time, under certain conditions of consumers are willing to and can afford to buy goods or services, here refers to the number of human medical institutions is still needed. When supply $>$ demand, supply exceeds demand, there will be graduates find jobs, when supply < demand, supply, medical institutions can have a large number of available jobs, is unable to provide satisfactory service, require higher medical colleges and universities to adjust professional structure, changing to adapt to the scale of its enrollment, medical institutions, the market of unit of choose and employ persons, when supply = the demand, the state is effective and satisfactory, it is kind of ideal state. Find out the gap between supply and demand, so as to clear the focal point of the development and utilization of human resources for health and training efforts should be made to the direction of the lead.

From the point of view of health manpower supply and demand, through the grey prediction method, for the next seven years, the medical higher education graduates to predict condition, compared with the health personnel demand forecasting results, it is concluded that the supply $>$ demand of 
human resources for health in china, according to "theory of supply and demand" in economics, the next few years there will be a large number of medical graduates can't find work, will cause serious waste of health talents.

In conclusion, in the next period, human resources for health should enlarge the number and size of medical students training, but should adapt to the health of the labor market demand and development of medical science and technology level, from adjust the training level, professional Settings, improve the overall quality of medical students, improving the quality and efficiency of training.

The cultivation of medical education is different from the other professional, medical students service object is a person in the future, life the first wealth is health, more rigorous training of medical students, enrollment expansion on not just the school buildings and logistics guarantee, more important is to have the guarantee of teachers, there are plenty of clinical practice teaching base. "Narrow into strict out" to guarantee the high quality personnel for health institutions.

With the development of social economy in our country, medical education structure unreasonable phenomenon is more prominent, the enrollment of medical colleges and training is not effective to adapt to the market economy rule of operation mode, the lack of health education on the management and effective coordination between planning department, medical colleges and universities enrollment scale, professional Settings, and so on can't well adapt to the needs of health care and reform, the unreasonable structure of the medical education, medical professional talent training in the field of coordinated development. Chinese medical service is far lower than the national requirements of medical level, the nurse and the insufficient medical technicians, management personnel to the low level of the status quo of the specialized institutions, prompt medical higher education, on the professional structure setting, at the same time, for the development of clinical related cannot ignore edge medicine and the development of cross subject. Except nurses and medical aspects of the relevant professional, general practitioners, public health professional such as preventive medicine and nutrition health professional training and the number of health service management and related professional training should also gradually to increase, so as to meet the health medical personnel planning of china and policy for the demand of the medical personnel.

\section{Comprehensive analysis of the relationship of medical \\ higher education and the health human resources, the supply and demand, has important practical significance.}

Medical personnel's overall quality is low. Medical market demands for medical personnel to the highly educated tilt. Therefore, medical education should give attention to both policy and market demand complementary principle, combined with the optimization level, specific as follows: further compressed the number and size of secondary health school, on the basis of moderate development of undergraduate education, appropriate control and compression specialist level of recruit students, on the premise of ensuring quality to accelerate the development of graduate education. Among them, college, technical secondary school level is given priority to withhold health technical education, slash and phasing out specialist level medical specialties. Medical specialties for undergraduate and graduate levels to give priority to, moderate speed up undergraduate and graduate level health technical professional development. Continue to maintain the medical basic research, applied research and study the proportion of talent training, focus on strengthening of preventive medicine, health service management applied to the cultivation of the postgraduate, gradually increase the proportion of graduate education in traditional Chinese. On-the-job and directed the cultivation of the postgraduate is widened, actively support and encourage outstanding young and middle-aged backbone of study for a higher degree. At the same time, our medical institutions will be actively encouraged on-the-job personnel further study.

The outstanding problem facing the human resources for health: health human resources more abundant but per thousand population health personnel have low unreasonable distribution. Overall quality of the health workforce is low. Human resources for health area configuration is not reasonable. Guangdong medical higher education the reality of the situation is: the recruitment of students scale is too large, growing too fast. Professional setting unreasonable training level is low. Health manpower demand > supply as a whole. For china human resources for health and medical institutions of higher education between the existing problems, and through the analysis of the preliminary draw the following planning proposal: the general principle of the development of human resources for health should be coordinated development, adjust structure, improve quality, expand the amount of health personnel, especially in medical and nursing staff number, efforts to improve per thousand population health workforce has several. Strengthen on-the-job training and continuing education; comprehensively improve the overall quality of health personnel. Strengthen the macroeconomic regulation and control; balance the medical personnel of regional development.

\section{CONCLUSION}

Eventually to achieve the purpose of this study is to government decision-making departments in formulating health workforce development planning and medical higher education development planning provides valuable scientific evidence, a target allocation of health resources, make the limited health resources to exert maximum efficiency, so as to better service for people's health.

\section{REFERENCES}

[1] Fleiszer DM, Posel NH, Stcacy SP. New direction in medicale-curricula and the of digital repositories. Acad Med. 2004 Mar; 79 (3):229-235.

[2] Kittidilokkul S, Tandcha roensathien V. Man powermix in private hospitals in Thailand: a census report [R].

[3] Herry R Mason. Manpower needsby specialty [J]. JAMA, 2004, 219, 1972:1621-1626.

[4] Chant AD. A confusion of roles: manpower in the National Health Service. J R. Soc. Med, 1998, 91: 63-65.

[5] Shipman SA, Liuri JD, Goodman DC. The general pediatrician: projecting future workforce supply and requirements [J]. Pediatrics, 2004, 113 (3): 435-442. 